Cahiers de recherches médiévales

\title{
César « d'ire enflamez et espris » (v. 1696)
}

dans le Roman de Jules César de Jean de Thuin

\section{Silvère Menegaldo}

\section{(2) OpenEdition \\ Journals}

Édition électronique

URL : https://journals.openedition.org/crm/848

DOI : $10.4000 / \mathrm{crm} .848$

ISSN : 1955-2424

Éditeur

Honoré Champion

Édition imprimée

Date de publication : 30 mars 2006

Pagination : $59-76$

ISSN : 1272-9752

Référence électronique

Silvère Menegaldo, «César " d'ire enflamez et espris » (v. 1696) », Cahiers de recherches médiévales [En ligne], 13 spécial | 2006, mis en ligne le 03 avril 2009, consulté le 15 décembre 2022. URL : http:// journals.openedition.org/crm/848; DOI : https://doi.org/10.4000/crm.848 


\title{
酷M
}

\author{
César « d'ire enflamez et espris » (v. 1696) \\ dans Le Roman de Jules César de Jean de Thuin
}

Du Roman de Jules César, œuvre peu connue et rarement visitée par la critique, on connaît aujourd'hui deux versions, l'une en prose, éditée voilà plus d'un siècle par F. Settegast et connue sous le titre de Hystore de Julius Cesar, à distinguer du Roman de Jules César proprement dit (dorénavant $R J C$ ), texte en vers édité beaucoup plus récemment par $\mathrm{O}$. Collet, qui lui a également consacré une étude étendue $^{1}$. Dans l'un et l'autre cas, il s'agit essentiellement d'une traduction - ou plutôt d'une adaptation, comme bien souvent au Moyen Âge - de La Guerre civile de Lucain (dorénavant $L G C$ ), complétée à la fin, puisque le poème épique est inachevé, par les trois ouvrages césariens rattachés aux Commentaires, le Bellum Alexandrinum, Africum et Hispaniense. Même si la chose n'est pas certaine, il est probable que les deux versions soient à attribuer au même auteur, un certain Jean de Thuin, et à dater des environs de $1260^{2}$; en outre, suite aux analyses de P. Hess, on peut considérer avec $\mathrm{O}$. Collet comme établi que la version en prose constitue un dérimage de la version en vers, dont elle ne diffère d'ailleurs pas de façon bien notable ${ }^{3}$.

Cette particularité assez remarquable, le fait qu'un même roman ait pris presque conjointement deux formes différentes, trouve peut-être mieux à s'expliquer si l'on considère le $R J C$ dans son contexte littéraire, proche et plus lointain.

Le contexte proche, c'est la première moitié du XIII ${ }^{\mathrm{e}}$ siècle, où la production historiographique en langue vulgaire et en prose manifeste un intérêt nouveau pour l'histoire romaine, avec l'Histoire ancienne jusqu'à César (qui parle en fait fort peu de César) et surtout les Faits des Romains, dont le projet initial était semble-t-il de produire un équivalent des Vies des douze Césars, mais qui s'est finalement limité à une biographie du seul César, en s'appuyant notamment sur les textes de Salluste, Suétone et César lui-même, mais aussi de Lucain ${ }^{4}$. Étant donné le choix de son sujet, dans la mesure en outre où Jean de Thuin avait certainement des Faits des Romains

\footnotetext{
${ }^{1}$ Voir Li Hystore de Julius Cesar, éd. F. Settegast, Halle, Niemeyer, 1881 ; Le Roman de Jules César, éd. O. Collet, Genève, Droz, 1993 ; O. Collet, Étude philologique et littéraire sur Le Roman de Jules César, Genève, Droz, 1993. On trouvera toute la bibliographie concernant les deux versions de notre roman dans ce dernier ouvrage, bibliographie qui ne s'est d'ailleurs à notre connnaissance guère accrue depuis 1993, sinon avec G.M. Spiegel, Romancing the Past. The Rise of Vernacular Prose Historiography in Thirteenth-Century France, University of California, 1993, p. 182-213 (sur la version en prose).

${ }^{2}$ Datation proposée par O. Collet dans son Étude, p. 76. En fait on ne peut situer les deux œuvres avec quelque certitude qu'entre 1212-1213 (date de rédaction des Faits des Romains) et 1276-1277 (celle d'un ms. reproduisant un passage de l'Hystore).

${ }^{3}$ Voir P. Hess, Li Roumanz de Julius Cesar. Ein Beitrag zur Caesargeschichte im Mittelalter, Winterthur, Keller, 1956, p. 20-26 et O. Collet, Étude, p. 92.

${ }^{4}$ Voir sur ces deux textes l'étude récente de C. Croizy-Naquet, Écrire l'histoire romaine au début du XIII siècle. L'Histoire ancienne jusqu'à César et les Faits des Romains, Paris, Champion, 1999.
}

Cahiers de Recherches Médiévales, 13spé, 2005 
une connaissance directe ${ }^{5}$, on peut donc considérer qu'il s'inscrit lui aussi dans ce mouvement historiographique porté vers l'histoire romaine, et vers une recherche de narration plus fidèle aux témoignages événementiels du passé, que caractérise notamment le recours à la prose; et en ce sens il se pourrait que le dérimage du $R J C$ signale la volonté de se conformer plus franchement à ce modèle, peut-être parce que le choix du vers ne s'est pas finalement révélé le plus approprié à son sujet, du moins à la date à laquelle le roman a été composé.

Néanmoins le $R J C$ est bien au départ un récit en vers, composé en laisses d'alexandrins, et qui tient à la fois de la chanson de geste et du roman, dans la mesure notamment où il adapte un texte latin. Aussi en tant que tel le RJC suit-il en premier lieu un autre modèle, plus romanesque qu'historiographique, et qui appartient à son contexte littéraire plus éloigné : c'est le modèle des romans dits «antiques » du XII ${ }^{\mathrm{e}}$ siècle, mais toujours très lus au XIII ${ }^{\mathrm{e}}$ siècle, qui s'offrait évidemment à un auteur médiéval prétendant adapter sous forme versifiée le poème de Lucain. Pendant tout le XII ${ }^{\mathrm{e}}$ siècle en effet, le roman médiéval se constitue à partir de la mise en roman, de la traduction et de l'adaptation de textes latins, depuis le Roman d'Alexandre d'Albéric de Pisançon, probablement le premier du genre (premier tiers du XII ${ }^{\mathrm{e}}$ siècle), jusqu'aux romans de Thomas de Kent ou d'Alexandre de Paris (vers 1180), en passant par le Roman d'Apollonius de Tyr, le Roman de Thèbes, le Roman d'Eneas et le Roman de Troie de Benoît de Sainte-Maure. Cependant, né de la traduction et de l'adaptation d'œuvres latines antiques ou plus tardives, assez rapidement, comme en témoigne l'évolution du genre alors en formation, le roman semble s'écarter irrémédiablement de la traduction pour glisser dans la seule adaptation (par exemple celle «d'un conte d'aventure », sans autre précision, comme le fait Chrétien de Troyes dans Erec et Enide) ou dans l'invention pure et simple, parfois d'ailleurs sous couvert de prétendue traduction, comme c'est le cas pour les romans de Hue de Rotelande ou le Florimont d'Aimon de Varennes. Comment expliquer cette évolution ? Est-ce l'extraordinaire succès du modèle romanesque arthurien forgé par Chrétien, popularisé par ses nombreux épigones, qui justifie l'abandon de la traduction? Pourtant on persiste jusqu'au début du XIII ${ }^{\mathrm{e}}$ siècle à composer des romans à cadre plus ou moins antique, comme Florimont ou Athis et Prophilias. Estce plutôt l'épuisement de la matière antique, comme pourrait le laisser entendre Marie de France, qui déclare dans le prologue des ses Lais avoir d'abord voulu traduire une histoire du latin, avant de renoncer puisque «itant s'en sunt altre entremis» ? C'est peut-être là une explication possible, la quantité de textes latins susceptibles d'être mis en roman n'étant pas inépuisable, certaines œuvres étant fort rares (Valerius Flaccus, par exemple). Mais ce n'était certainement pas le cas de Lucain, qui a été l'un des auteurs antiques les plus lus dès le début du Moyen Âge, comme en témoigne le nombre de mss. conservés (plus de deux cents mss. et fragments de $L G C$ entre le $\mathrm{IX}^{\mathrm{e}}$ et le $\mathrm{XII}^{\mathrm{e}}$ siècle, selon le relevé de $\mathrm{B}$. Munk Olsen ${ }^{6}$, soit plus que de L'Énéide à la même période), l'abondance des scholies, des accessus et

\footnotetext{
${ }^{5}$ Sur les liens évidents entre notre roman et les Faits des Romains, avec une série de rapprochements probants, voir O. Collet, Étude, p. 187-189.

${ }^{6}$ Voir B. Munk Olsen, L'Étude des auteurs classiques latins aux XI et XII ${ }^{e}$ siècles, CNRS, 1982-1987, tome deux. De la même période, on ne connaît qu'un seul ms. complet des Argonautiques de Valerius Flaccus.
} 
des commentaires, des citations et des réminiscences dans toutes sortes de textes, en latin comme en langue vulgaire ${ }^{7}$.

D'où la question au départ de cette étude, eu égard au succès dont bénéficiait le poète latin dès le XII ${ }^{\mathrm{e}}$ siècle : pourquoi a-t-il fallu attendre si longtemps, plus d'un siècle, pour qu'un adaptateur se décide à metre en roman l'épopée de Lucain, à la manière du Roman de Thèbes adaptant Stace? Si on laisse de côté des explications toujours envisageables mais forcément peu satisfaisantes comme le hasard ou une traduction antérieure perdue, quels sont les facteurs à même d'expliquer un tel délai, soit dans le texte même de $L G C$, soit dans sa réception médiévale, soit dans des éléments extérieurs à Lucain lui-même et liés par exemple à l'image médiévale de César?

On peut certainement avancer diverses hypothèses pour expliquer cet étonnant retard dans l'adaptation romanesque de Lucain ${ }^{8}$, ce qui ne va pas d'ailleurs sans conférer au RJC le statut d'objet littéraire un peu obsolète. Parmi elles cependant, il nous semble que la principale pourrait bien tenir à la figure même de César, particulièrement malmenée chez Lucain, et aux difficultés de traitement qu'elle pouvait poser à un adaptateur médiéval, dont rend bien compte à sa manière le travail de Jean de Thuin. C'est donc au personnage de César dans le RJC que nous nous attacherons essentiellement dans les pages qui suivent, sans perdre toutefois de vue l'interrogation au départ de notre réflexion.

\section{César en héros chevaleresque dans le Roman de Jules César}

Avant tout il faut préciser que le $R J C$ n'est pas à proprement parler, ne peut pas être, étant donné les pratiques médiévales encore en usage au XIII ${ }^{\mathrm{e}}$ siècle en cette matière, une traduction de $L G C$ de Lucain, et ne prétend d'ailleurs pas en être

\footnotetext{
${ }^{7}$ De nombreux travaux ont été consacrés à la réception de Lucain au Moyen Âge. Sans prétendre à l'exhaustivité, il faut au moins ajouter à la liste déjà fournie d'études citées par O. Collet (Étude, p. 90) deux articles importants de P. von Moos, "Poeta und historicus im Mittelalter. Zum Mimesis-Problem am Beispiel einiger Urteile über Lucan», Beiträge zur Geschichte der deutschen Sprache und Literatur, Tübingen, 98, 1976, p. 93-130, ainsi que «Lucans tragedia im Hochmittelalter. Pessimismus, contemptus mundi und Gegenwartserfahrung », Mittellateinisches Jahrbuch, 14, 1979, p. 127-186 ; voir aussi la précieuse mise au point introductive, appuyée sur une abondante bibliographie, d'E. d'Angelo dans «La Pharsalia nell'epica latina medievale », Interpretare Lucano. Miscellanea di studi, éd. P. Esposito et L. Nicastri, Université de Salerne, Naples, 1999, p. 389-453 ; en revanche nous n'avons pu consulter S. Werner, The Transmission and Scholia to Lucan's Bellum civile, LIT, Münster, 1998. Il existe enfin de très nombreux articles sur la réception de Lucain dans telle ou telle œuvre médiévale (en particulier dans celle de Dante).

${ }^{8}$ À titre d'exemple, on peut invoquer les problèmes que pouvait poser le texte de Lucain, non seulement à cause de sa difficulté, mais surtout à cause de son inachèvement. Pour compléter $L G C$ il fallait nécessairement avoir recours à d'autres textes, notamment ceux du corpus césarien, qui étaient connus au XII ${ }^{\mathrm{e}}$ siècle, mais beaucoup moins faciles d'accès : en effet, parmi la trentaine de mss. contenant des œuvres de César recensés par B. Munk Olsen entre le $\mathrm{IX}^{\mathrm{e}}$ et le XII ${ }^{\mathrm{e}}$ siècle, seuls neuf sont complets et comprennent l'ensemble du corpus. Mais ce n'est là qu'une explication parmi d'autres, et nous tâcherons dans un prochain article de les envisager de façon plus complète.
} 
une: l'auteur se contente d'évoquer dans son prologue une «matere qui n'est pas moult oïe » qu'il va du latin changier en roman, le nom de Lucain n'apparaissant pas avant la première rubrique annonçant «Le premier livre de Lucan». Il s'agit donc, comme le rappelle $\mathrm{O}$. Collet ${ }^{9}$, d'adapter autant que de traduire $L G C$, ce qui implique de faire subir au texte latin un certain nombre de transformations, pour ne pas dire de gauchissements. La question ayant déjà été bien étudiée ${ }^{10}$, il ne nous apparait pas utile de revenir sur une comparaison de détail entre le roman de Jean de Thuin et l'œuvre de Lucain. Il suffit de noter que dans l'ensemble le RJC se comporte vis-àvis de sa source latine à peu près comme les romans antiques du XII ${ }^{\mathrm{e}}$ siècle vis-à-vis de la leur, du moins ceux qui lui sont comparables dans la mesure où ils s'appuient exclusivement sur un texte, comme le Roman d'Eneas et le Roman de Thèbes. Ce qui veut dire notamment ${ }^{11}$ : un respect global de la structure narrative du texte source, sans que les auteurs s'interdisent néanmoins de supprimer, d'ajouter, de réduire ou de développer certains épisodes (ainsi celui des amours entre César et Cléopâtre dans le $R J C$ ) ; la suppression presque systématique de certains éléments, liés par exemple à la religion ou à la mythologie antiques, aux développements savants (géographiques, onomastiques etc.) ou encore au surnaturel (dont le $R J C$, c'est à noter, est totalement dépourvu); la transposition globale des réalités sociales, politiques, militaires de l'Antiquité dans le monde médiéval contemporain des auteurs, ce qui aboutit parfois, dans le $R J C$, à des simplifications ou à des gauchissements plus ou moins importants ${ }^{12}$.

César, de même que les autres personnages de $L G C$, plus qu'eux encore, n'est pas épargné par ce travail d'adaptation. On sait en effet que dans le poème de Lucain le général romain apparaît sous un jour particulièrement défavorable, quand bien même on trouverait quelques indices allant dans un sens plus flatteur; et que, cause et conséquence à la fois de ce traitement, il n'est certainement pas le héros de $L G C$, dans un récit construit au contraire de manière à faire alterner et à opposer diverses figures de premier plan, essentiellement César, Pompée et Caton ${ }^{13}$. Or la perspective adoptée par Jean de Thuin est bien différente: non seulement dans le $R J C$, comme son titre l'indique, César est bel et bien le héros, Pompée et Caton

\footnotetext{
${ }^{9}$ O. Collet, Étude, p. 95-96.

${ }^{10}$ Pour le RJC, voir O. Collet, Étude, notamment p. 155-177, que l'on peut compléter, mais cette fois à propos de la version en prose, par V.L. Dedecek, Étude littéraire et linguistique de Li Hystore de Julius Cesar de Jehan de Tuim, Paris, Champion, 1925.

${ }^{11}$ Sur ces questions, abondamment étudiées, on peut toujours se reporter au troisième chapitre d'A. Petit, Naissances du roman. Les techniques littéraires dans les romans antiques du XII siècle, Paris, Champion, 1985.

${ }^{12}$ Comme le montre $\mathrm{O}$. Collet, le $R J C$ peine à rendre les spécificités politiques et historiques du monde romain en général et de la guerre civile en particulier, même s'il est parfois plus précis qu'on pourrait le penser (voir sur ce point les p. 162-165). C'est un point qui mérite d'être noté, non qu'il soit forcément signe d'ignorance ou d'incompréhension, mais qui permet de souligner la différence entre un texte à vocation romanesque comme le $R J C$ d'un texte à vocation historique comme les Faits des Romains, en l'occurrence beaucoup plus attentif à la restitution et à l'explication des réalités romaines.

${ }^{13}$ Pour une mise au point récente sur la figure de César dans $L G C$, voir L. Nosarti, «Quale Cesare in Lucano ? », Acta classica Universitatis Scientiarum Debreceniensis, 38-39, 20022003, p. 169-203.
} 
passant au second plan, mais surtout il bénéficie d'une réévaluation dans un sens nettement plus favorable, ce qui n'était pas une mince gageure avec Lucain pour point de départ ${ }^{14}$.

Considérons un peu plus en détail les éléments de cette revalorisation, en nous intéressant d'abord aux critères proprement hiérarchiques, qui font de César le héros, puis, même s'ils sont étroitement liés aux premiers, aux critères évaluatifs, qui permettent de le revaloriser.

D'un point de vue purement quantitatif, cela ne fait aucun doute, César est bien le personnage principal du $R J C$, presque toujours présent du début à la fin du roman, qui s'ouvre sur le franchissement du Rubicon et s'achève sur son entrée triomphale dans Rome et son couronnement comme empereur (avant son assassinat, donc, qui n'est pas évoqué dans le RJC, au contraire des Faits des Romains), établissant ainsi une progression depuis le triomphe refusé au retour des Gaules au triomphe couronnant la fin de la guerre civile. Cette mise au premier plan du personnage de César est d'ailleurs annoncée d'emblée, dès le prologue, où l'auteur dit son intention de raconter

l'estoire des Romains et por quoi et comment

Julis Cesar li preus qui tant ot hardement

la guerre commença et mena longuement

vers les citains de Rome qu'il par esforcement enz es chans de Tessale desconfi plainement, et qui par sa valor se l'estoire ne ment citez, bors et chastiaus conquist si amplement com li cieux le mont coevre et la terre s'estent. (v. 4-11)

Le programme du roman, d'ailleurs réitéré à la laisse suivante, c'est donc d'abord César et ses conquêtes ( «l'emperor Cesar qui par sa baronnie / le plus du mont conquist et mist en sa baillie», dit le début de la laisse 2), César dont la «bonté» (autrement dit le «mérite», la «vertu») doit constituer pour tout «haus hom » un «example et bon enseignement» (v. 17), César en somme plus que la guerre civile, plus que «l'estoire des Romains » (à laquelle le parcours césarien, au v. 4, semble en quelque sorte s'assimiler ou se substituer) - programme dont le portrait final de Jules en empereur (laisse 488) se charge de confirmer la réalisation.

Conséquence de cette nouvelle hiérarchie dans le personnel romanesque, tous les autres personnages passent plus ou moins au second plan, y compris Pompée et Caton. En outre, tandis que Caton conserve un traitement très favorable, Pompée connaît une évolution qui le constitue nettement en opposant du héros césarien, notamment par l'imputation d'un crime totalement étranger à l'œuvre de Lucain (et pour cause), celui d'avoir offensé le Dieu des Chrétiens en se servant du Temple de Salomon comme étable : «Et Diex li fist si bien cest forfait meriter / que a Cesar le

${ }^{14}$ Sur le personnage de César dans le RJC, voir surtout O. Collet, Étude, en particulier p. 102, 106-110,141-142, 149, 174 et 194. En ce qui concerne l'Hystore, on pourra se reportera à G. Spiegel, Romancing the Past, p. 182-213 et à J. Beer, «Jean de Tuim's Julius Caesar : a model of 'bonté' for the thirteenth century », Mélanges Jean Dufournet, Paris, Champion, 1993, p. 175-182. 
fist en bataille mater»(v. 1408-1409) ${ }^{15}$. Ainsi, par opposition à Pompée, César, instrument de la vengeance divine (voir aussi la laisse 119), se trouve en quelque sorte implicitement christianisé, et donc valorisé, dans la perspective médiévale. C'est d'ailleurs par un procédé similaire, tout aussi audacieux et tout aussi anachronique, que César se trouve en quelque sorte assimilé au domaine français, et donc marqué d'un signe positif (dans la perspective d'un auteur écrivant en français au XIII ${ }^{\mathrm{e}}$ siècle, bien sûr) qui le constitue clairement en héros : au moment de son entrée en Italie, Jean de Thuin compare en effet le général romain à Charlemagne, étant donné le nombre de soldats qu'il a pu rassembler, dont par ailleurs beaucoup lui sont venus de «France » $(\text { v. } 781)^{16}$; de même Pompée dans $L G C$ parle à propos de César de la gallica rabies qui se répand à travers les Alpes ${ }^{17}$, mais dans la perspective romaine cette assimilation aux Gaulois prend évidemment un sens bien différent.

En même temps qu'il accède au statut de héros, César se trouve doté de multiples qualités, résultant à la fois de ce que Jean de Thuin pouvait trouver chez Lucain (où César n'en apparaît pas totalement dépourvu) et de la transposition dans l'univers médiéval du texte latin, faisant du général romain un véritable héros chevaleresque. Parmi toutes ces qualités, ce sont avant tout, de façon très attendue, les vertus guerrières et militaires qui apparaissent mises en avant, notamment par le jeu des épithètes de caractère (en position détachée par l'article défini), telles César $l i$ preus (v. 5, 1496, 4054 etc.), li combatanz (v. 115, 156, 7343 etc.), li ber (v. 728, 2987, 3362 etc.) ou li vaillans (v. 728, 1039, 2506 etc.), formules stéréotypées disant aussi de façon plus générale la valeur de César, comme d'autres qui le sont moins (voir v. 390, 871, 1463-1464 etc.). Et les actions du personnage s'adaptent à ces qualifications, puisqu'on voit à plusieurs reprises dans le récit, lors de telle ou telle bataille, César se battre avec la plus grande vaillance et se comporter comme le meilleur des chevaliers, par exemple lors du siège de Lerida (laisses 114-118) ou lors des batailles de Pharsale (laisses 255-257) ou de Thapsus (laisses 460-463). C'est d'ailleurs dans cette dernière bataille que la transformation de César en héros de roman apparaît la plus évidente, à l'occasion d'un véritable portrait de l'empereur romain en chevalier médiéval :

Cesar li ber seoit desor .I. auferrant,

.I. bon destrier d'Espaigne qui tost l'en vait portant,

et .I. moult riche escu ot a son col pendant

qui toz iert pains a or esmeré et parant,

et ou lieu ou la boucle doit estre par devant,

la avoit .I. lion a sa bouche baant

entaillié tout de sable a guise bien seant.

[Ces haubers fut toz fais d'erjant resplandoiant

a maille antrelacie menue et bien joingnant],

\footnotetext{
${ }^{15}$ Sur cet élément, qui n'est d'ailleurs pas propre au RJC, puisqu'on le trouve aussi dans les Faits des Romains, voir O. Collet, Étude, p. 107-108.

${ }^{16}$ Jean de Thuin se souviendra de ce point quand il évoquera bien plus tard les exploits des trente chevaliers «qui de France erent né » (v. 7975) à la bataille de Thapsus.

${ }^{17}$ Lucain, La Guerre civile, éd. et trad. A. Bourgery et M. Ponchont, Paris, Les Belles Lettres, 1927-1930, Livre II, v. 535.
} 


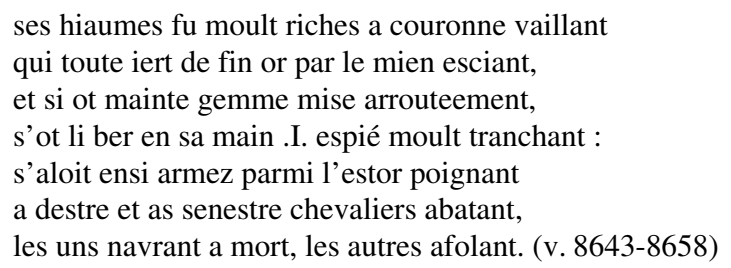

On retrouve ici tous les attributs du chevalier médiéval, cheval, armes et armoiries comprises (qui renvoient bien sûr métaphoriquement à César lui-même, ailleurs très traditionnellement comparé à un «lyon», v. 2521), et surtout la vaillance au combat qui lui fait abattre une multitude d'ennemis.

Toujours guerrières, mais peut-être moins attendues dans un roman médiéval, et partant plus spécifiques au personnage de César, et aussi au texte de Lucain, où on en trouve diverses traces: ses qualités de chef militaire, de stratège, soulignées notamment lors des sièges de Marseille, Brundisium, Lerida ou Dyrrachium (c'est à ce propos qu'il est question de l'engin de César, par exemple v. 1245 et 2229); son art d'ordener ses troupes sur un champ de bataille (v. 4236, 7875, 8054 etc.) ; ses qualités de meneur d'hommes, qui sait ennorter, conforter ou semondre ses soldats, comme on le voit en particulier à la bataille de Pharsale (voir les laisses 236-261, mais aussi ailleurs, v. 7502, 8069 ou 8590-8591).

Enfin le spectre s'élargit encore avec les qualités suivantes, plus directement, ou plus du tout guerrières, mais qui n'en sont pas moins encore celles que l'on peut attendre d'un héros de roman: la pité, la douçor ou la debonnereté (v. 338, 1072, 1378 ou 7743), autrement dit la clémence ${ }^{18}$, illustrée à diverses occasions, notamment à Corfinium (laisse 59), à Brundisium (laisse 75), à Lerida (laisse 142) ou encore à Alexandrie (laisse 422) ; la largesce (v. 6892), qui consiste à distribuer généreusement argent et terres à ses partisans (voir notamment les laisses 268, 431, 471 et 487) ; enfin les qualités courtoises et amoureuses, dont il se trouve pourvu grâce au développement et à la réinterprétation de l'épisode de Cléopâtre : pour ces dernières cependant, le fait est à souligner, ce n'est pas le narrateur qui lui prête, mais le chevalier que César lui-même a envoyé en ambassade auprès de Cléopâtre, et qui fait le portrait le plus flatteur du général romain, «biax et cortois et si preuz que trover / ou remenant du monde ne porroit on son per», «miex letré ne plus sage ne porroit on trover / et avec ce molt a large cuer por doner» (v. 6886-6890).

\footnotetext{
${ }^{18}$ La fameuse clémence césarienne, si malmenée par Lucain, apparaît à nouveau comme l'une des qualités du personnage dans le RJC. Affirmer néanmoins, comme le fait J. Beer (art. cit.), que Jean de Thuin, du moins dans la version en prose de son roman, en fait la qualité essentielle de César nous paraît pour le moins excessif, surtout que cette interprétation doit reposer en partie sur un contresens : en effet la bonté dont il est question dans l'Hystore, et notamment dans le prologue (p. 2, 1. 13), ne désigne probablement pas précisément la «clémence » de César, mais de façon beaucoup plus générale sa «vertu» ou son «mérite», comme dans le $R J C$ d'ailleurs.
} 


\section{César «d'ire enflamez et espris » (v. 1696)}

Ce serait plus qu'assez pour faire de César le parfait héros de roman, si on ne trouvait pas par ailleurs dans le $R J C$ des qualifications beaucoup plus ambiguës, voire franchement péjoratives, qu'il nous semble possible de ranger sous trois rubriques :

1) il faut d'abord mentionner l'orgueil, même si dans le texte ce reproche est presque systématiquement adressé à César par ses ennemis (v. 1148, par Pompée; v. 4662, par Domitius ; v. 5522, par Caton ; v. 8610, par Scipion ; v. 9066, par les partisans de Caton), et rarement pris en charge par le narrateur, sauf à deux reprises (v. 379, dans un passage d'ailleurs plutôt embrouillé, et v. 6310, quand l'orgueil de César est vaincu par l'amour de Cléopâtre). Ce n'est donc pas ce défaut, de toute manière inhérent à la figure du conquérant, comme Alexandre peut en témoigner au Moyen Âge, qui se trouve particulièrement souligné, et il ne l'est d'ailleurs pas non plus dans le texte de Lucain ${ }^{19}$.

2) signalée à diverses reprises, la fierté de César apparaît plutôt ambiguë (conformément d'ailleurs aux acceptions médiévales du terme) : parfois «fierté, courage » (voir par exemple les v. 336, 3241, 6310 etc.), mais plus souvent «férocité, cruauté » (notamment v. 1006 et 1033, v. 2320, 2403 et 2521, ou encore v. 2990, lorsque César mate impitoyablement une révolte dans son armée) ; Caton fait d'ailleurs reproche à son adversaire de sa «grant cruialté » (v. 5522), dont on a un bel exemple en voyant à la fin du roman César le «cuer esbaudi et joiant» (v. 8948) de ses succès, sans accorder la moindre pensée aux morts dont il est responsable.

3) reste enfin un élément qui par ses mentions réitérées occupe dans le $R J C$ une place à part, beaucoup plus importante que les deux précédents, c'est l'ire, la rage, le maltalent, le malgré de César (voir en particulier, parmi les occurrences les plus significatives de ces différents substantifs et de leurs dérivés verbaux ou adjectivaux: v. 401, 426, 1060, 1334, 1435, 1597 et suivants, 1645 et suivants, 1696 et suivants, 2043, 2988, 2512, 4507, 4616, 7751, 7857). D'abord explicitement liées, au début du roman, au refus du triomphe, ces manifestations de colère ou de rage se multiplient ensuite et finissent par constituer l'un des traits les plus saillants du caractère de César. Quelle portée prêter à cette ire qui ne cesse d'animer le général romain ? Elle est certainement à rapprocher selon nous d'un autre trait permanent du caractère césarien, son goût de la guerre, ce qu'on pourrait appeler son bellicisme, également souligné à diverses reprises, notamment à l'occasion de cette belle formule évoquant un «Cesar qui volage / avoit le cuer viers guerre et vers pais moult ombrage » (v. 605-606; voir aussi les v. 858, 3099, 3108 ou 3274-3276). Comme on s'en rend particulièrement compte à l'occasion de la bataille de Pharsale, l'ire césarienne nous paraît donc surtout correspondre, en tant que trait permanent de caractère et non en tant que manifestation ponctuelle, à une sorte de rage ou de frénésie guerrière, qui ne trouve à se satisfaire que dans la violence de la bataille.

\footnotetext{
${ }^{19}$ Pour le défaut d'orgueil, il est plus probable de supposer l'influence des Faits des Romains (qui en l'occurrence s'inspirent de Suétone), où ce point est objet de développements particuliers dans le portrait final consacré à César. Dans la mesure cependant où ce défaut n'apparaît ainsi attaché à César qu'à la fin de sa vie, il faut peut-être voir dans le $R J C$ une autre influence, celle du Roman d'Alexandre par exemple.
} 
Cette particularité, il faut le souligner, n'est pas de l'invention de Jean de Thuin, mais trouve son exact correspondant dans $L G C$, où, comme le rappelle $\mathrm{L}$. Nosarti, «l'ira è una costante in tutte le azioni di Cesare $»^{20}$, mentionnée qu'elle est à propos du général romain dès le portrait liminaire du Livre I (v. 146), puis à d'innombrables reprises dans la suite du poème - et notamment dans ce vers fameux du Livre VII, lors de la bataille de Pharsale, «Hic furor, hic rabies, hic sunt tua crimina, Caesar»(v. 551). Ce qui apparaît clairement dans la perspective stoïcienne (rappelons le De ira de Sénèque) de Lucain comme une passion inutile, dangereuse et même destructrice dans le cas de César, et peut-être finalement comme la passion qui se trouve au fondement du portrait exclusivement défavorable du général romain $^{21}$, n'a donc pas disparu du $R J C$ et continue de marquer le personnage d'une empreinte fortement négative. Au Moyen Âge en effet, si la colère (juste et mesurée) peut parfois prendre forme vertueuse, elle n'en reste pas moins avant tout un vice, et un vice capital ${ }^{22}$. L'ire césarienne, disposition permanente de l'esprit et non éruption momentanée, quand bien même elle serait (comme chez certains personnages épiques) la passion qui anime la vaillance et l'esprit de conquête du général romain, cette ire doit donc paraître avant tout condamner le personnage aux yeux d'un lecteur médiéval. En outre la colère dirige à ce point les actions de César qu'elle en vient parfois à remettre en cause certaines de ses qualités, et notamment sa clémence. Ainsi, dès le début du RJC, ulcéré par le triomphe qui lui a été refusé, on le voit déclarer: «En armes mosterrai vers elz m'umelité, / en m'espee metrai ma douçor, ma pité !» (v.337-338). De même à la bataille de Pharsale il exhorte ses hommes «que de nului n'eüssent ne merci ne pité / ne pour leur connaissance ne pour leur parenté »(v.4580-4581). Et c'est d'ailleurs au même moment, dans la même laisse 261, que le narrateur non seulement souligne l'horreur d'un combat opposant le père au fils et le frère au frère, mais condamne explicitement l'iror et le maltalent ayant abouti à une telle bataille, donc indirectement César lui-même :

${ }^{20}$ L. Nosarti, art. cit., p. 190. De même O. Steen Due (dans «Lucain et la philosophie», Lucain, éd. M. Durry, Genève, Fondation Hardt, Entretiens sur l'Antiquité classique 15, 1970, p. 211) affirme que « la passion la plus dominante dans le poème de Lucain, c'est la colère ». Une thèse de 1994 de la Johns Hopkins University (que nous n'avons pas consultée) a même été consacrée à ce sujet : A.E. Pierce, Omnia Caesar erat : the Power of ira in Lucan's Bellum civile.

${ }^{21}$ Voir les analyses de R. Glaesser dans Verbrechen und Verblendung. Untersuchung zum Furor-Begriff bei Lucan mit Berücksichtigung der Tragödien Senecas, Bern, Peter Lang, 1984, en particulier p. 18-71. Dans ces pages l'auteur développe une réflexion sur l'ira comme manifestation de la furor qui anime sans cesse César et qui en fait dans $L G C$ un «furor-Dämon », une sorte de génie de la destruction.

${ }^{22}$ Voir C. Casagrande et S. Vecchio, Histoire des péchés capitaux au Moyen Âge, Paris, Aubier, 2002, p. 94-125. L'ire au Moyen Âge fait d'abord partie des sept péchés capitaux, en tant que telle forcément condamnable. Cette condamnation néanmoins ne semble pas absolue, et l'éventualité existe d'un recours utile, sinon exactement vertueux, à la colère, en particulier dans les domaines militaire et politique (voir sur ce point Anger's Past. The Social Uses of an Emotion in the Middle Ages, éd. B.H. Rosenwein, Cornell University, 1998, en particulier les chapitres 3, 6 et 7). Il n'est donc pas impossible que Jean de Thuin entende ainsi, au moins en partie, l'ire césarienne, mais cela n'apparait jamais explicitement. 


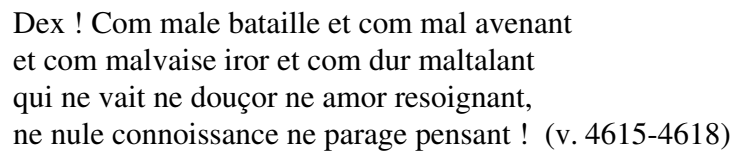

Le passage n'apparaît pas tel quel dans $L G C$, mais on peut supposer en fait qu'il en reprend un autre qui se trouve non pas à la fin mais au milieu de la bataille de Pharsale, au Livre VII : c'est justement celui où se trouve le vers que nous citions plus haut, «Hic furor, hic rabies, hic sunt tua crimina, Caesar», condamnation de César la plus violente peut-être parmi toutes celles qu'exprime Lucain. Ainsi l'ira césarienne, bien loin d'avoir disparu du RJC, continue de caractériser spécifiquement César, et de fournir le départ d'un jugement qui peut être globalement négatif sur le personnage, tel du moins qu'il est exprimé, même de façon voilée, à l'occassion de la bataille de Pharsale.

\section{Du César de Lucain au César de l'Hystore de Julius Cesar}

Malgré toutes les qualités dont il est pourvu, César apparaît donc marqué d'un certain nombre de traits ambigus, voire franchement dévalorisants, et en particulier de cette ire, de cette rage guerrière qui constitue déjà chez Lucain l'un de ses aspects les plus négatifs. Peut-être ces quelques traits sembleront-ils peu de choses, eu égard à la revalorisation globale dont bénéficie le personnage, traité de façon particulièrement défavorable dans $L G C$. Il apparaît pourtant, si l'on considère maintenant le $R J C$ dans son contexte littéraire médiéval, que cette revalorisation n'est pas forcément si importante, dans la mesure où elle était en quelque sorte inévitable étant donné le processus d'adaptation auquel le texte de Lucain était, et avait déjà été (dans les Faits des Romains), soumis ; que cette revalorisation est somme toute moins remarquable que la persistance chez César d'aspects négatifs, quels que soient les textes qui le mettent en scène.

Étant donné en effet le travail d'adaptation exercé par Jean de Thuin sur le texte de Lucain, comparable, on l'a dit, à celui des auteurs de romans antiques au $\mathrm{XII}^{\mathrm{e}}$ siècle, l'évolution du personnage de César apparaît d'abord comme un phénomène en quelque sorte nécessaire et attendu, et non pas forcément recherché par l'auteur, une revalorisation certes, mais pas une réhabilitation. Transposé d'un contexte culturel et littéraire latin, précisément celui de l'épopée impériale, à un contexte culturel et littéraire médiéval disons épico-romanesque, le personnage subit des évolutions allant nécessairement dans un sens plus ou moins favorable, où l'on peut distinguer l'action conjuguée de quatre facteurs :

1) d'abord et avant tout le facteur d'adaptation (ou de traduction-adaptation), déjà évoqué plus haut, qui suffit à expliquer pour une bonne part les modifications favorables apportées à César, par la simple transposition de ce personnage dans le contexte médiéval. Déjà les Faits des Romains, dont le projet est pourtant bien plus historique que romanesque, peuvent témoigner de ce phénomène, comme l'a montré 
notamment $\mathrm{J}$. Beer ${ }^{23}$; il est d'autant plus marqué dans le $R J C$, où le général romain devient un véritable héros romanesque.

2) ensuite le facteur générique, dans la mesure où le $R J C$, par son contenu et sa forme même (la laisse d'alexandrins) adopte clairement un modèle à la fois épique (on le voit surtout dans les récits de batailles) et romanesque (développement de l'épisode amoureux entre César et Cléopâtre), dont le représentant le plus évident est le Roman d'Alexandre (ne serait-ce que par sa forme), mais aussi les autres romans antiques du XII ${ }^{\mathrm{e}}$ siècle. Coulée dans un moule épico-romanesque médiéval, la figure de César est amenée nécessairement à prendre une tournure particulièrement héroïque qui ne correspond ni au modèle épique impérial de Lucain, ni au modèle plus historique des Faits des romains.

3) étroitement lié au précédent, le facteur hiérarchique qui fait de César le personnage central, le héros d'un récit relatant son ascension jusqu'au pouvoir impérial, et qui s'achève significativement avant sa mort. En abandonnant son personnage au sommet de la gloire, Jean de Thuin développe une structure narrative qui rappelle cette fois le roman arthurien de Chrétien de Troyes ou de ses épigones, bien différente là encore de la perspective nettement biographique adoptée par les Faits des Romains (ou même le Roman d'Alexandre) et qui fait nécessairement porter un autre éclairage sur le personnage.

4) reste enfin le facteur évaluatif proprement dit, dans lequel on peut ranger toutes les qualifications, tous les jugements favorables appliqués au personnage, qui relèvent explicitement d'une revalorisation effectuée par le narrateur. Dans le RJC cependant, c'est ce facteur qui nous semble jouer le moins, et c'est ce qui nous permet de penser que la réévalutation de César ne relève pas tant d'un choix d'auteur que d'une logique d'adaptation et de transposition générique. Il n'est que de considérer par exemple le sort des épithètes de caractère appliquées à César (César li ber, li preus etc.), assez typiques de l'écriture épique, qui disparaissent toutes sans exception de la version en prose du roman, ce qui tendrait à laisser croire que leur présence dans le $R J C$ est d'abord liée au facteur générique, le choix d'un modèle épicoromanesque (choix qui n'est plus celui du dérimage, où l'adoption de la prose signale probablement le retour à un modèle plus historique), plutôt qu'au facteur évaluatif proprement dit, impliquant une réelle volonté de revalorisation de la part de Jean de Thuin.

Ainsi il nous semble que c'est au processus d'adaptation qui s'exerce sur le personnage bien plus qu'à une intention affichée de réhabilitation qu'il faut imputer la revalorisation dont César est l'objet, revalorisation qui laisse de toute manière subsister d'importants aspects négatifs. On peut d'ailleurs aboutir au même constat, mais d'un autre point de vue, si l'on considère cette fois l'évolution que présente le $R J C$ non plus par rapport à Lucain, mais par rapport à la suite de trois textes (les Faits des Romains, le RJC en vers et sa version en prose, l'Hystore de Julius Cesar) dans laquelle il faut aussi le situer pour en comprendre les modalités. D'un texte à l'autre en effet, César apparaît l'objet d'une revalorisation toujours un peu plus marquée, sans jamais aller toutefois jusqu'à la réhabilitation complète. Ce n'est

\footnotetext{
${ }^{23}$ Voir J. Beer, A Medieval Caesar, Genève, Droz, 1976, les chapitres 8, 9 et 10, où l'auteur montre bien la revalorisation dont César est l'objet dans les Faits des Romains, par le seul fait de la transposition.
} 
guère surprenant dans la mesure où le $R J C$ comme l'Hystore s'appuient certes sur Lucain, mais aussi sur l'adaptation de Lucain qui les a précédés, et qui a déjà effectué un travail plus ou moins important dans le sens de la revalorisation : des Faits des Romains, dont Jean de Thuin s'est très certainement inspiré, au RJC, puis du $R J C$ à l'Hystore, César apparaît donc assez logiquement sous un jour de plus en plus favorable, mais sans jamais se départir complètement, ce qui est plus surprenant, de ses traits négatifs. De fait, dès les Faits des Romains, où $L G C$ n'est qu'une source parmi d'autres, le travail de l'adaptation permet de donner du personnage une vision bien plus favorable que celle de Lucain. Néanmoins, comme l'a bien montré C. Croizy-Naquet (en faisant en quelque sorte la synthèse des analyses plutôt contradictoires de J. Beer et de G. Spiegel sur cette question ${ }^{24}$ ), la représentation de César élaborée dans les Faits des Romains apparaît avant tout complexe et ambiguë :

Le portrait de César est travaillé dans ses divers aspects ; le héros est ainsi un être ambivalent en qui sont figurées les forces et les faiblesses de l'Homme. Ce personnage modèle, en ce qu'il détient les trois fonctions de prouesse, sagesse et richesse en biens et en plaisirs, est un être bi-frons : c'est un chef de guerre exemplaire, mais qui se compromet pour parvenir à ses fins ; c'est un homme de science, mais qui a la prétention de se mesurer à un dieu pour percer tous les secrets de la nature ; c'est un homme de désir, mais qui recherche les plaisirs exclusivement sexuels. ${ }^{25}$

Dans un second temps, des Faits des Romains au RJC, c'est évidemment le facteur générique qui joue le plus, avec le passage d'un récit à portée plutôt historique à un texte épico-romanesque. Il faut souligner d'ailleurs que dans cette mesure le RJC apparaît s'écrire autant avec que contre les Faits des Romains, entendant peut-être revenir à une plus grande proximité avec le modèle épique de Lucain. Ainsi le personnage de César est-il encore revalorisé, sans toutefois perdre ses traits négatifs, comme le rappelle d'ailleurs O. Collet à la fin de son Étude (p. 194), même si ce n'est pas ce que le critique veut d'abord souligner :

Le héros de notre récit, taillé au format des canons épiques et romanesques, s'avère peut-être imbu de pouvoir, jaloux, belliqueux, et démesurément orgueilleux, l'incarnation d'une force asservissante, dont l'action menace tous ceux qui s'y opposent, il n'en revêt pas moins une apparence très positive dans l'ensemble - chef désigné par son intelligence stratégique, son engin, par son aptitude au commandement militaire, par sa baronnie, sa vaillance et son endurance au combat, réunissant de surcroît les principales qualités, aristocratiques, courtoises, morales et politiques (générosité, mesure dans les actes, pardon, disponibilité envers l'amour etc.).

Enfin, du vers à la prose, du RJC à l'Hystore, le processus se poursuit, renforçant encore la réévaluation dont bénéficie César, mais encore une fois sans effacer les

\footnotetext{
${ }^{24}$ Dans son étude déjà citée, A Medieval Caesar, J. Beer insiste surtout sur la revalorisation dont César est l'objet dans les Faits des Romains, au contraire de G. Spiegel (dans Romancing the Past, p. 159-182), qui considère que la représentation du général romain reste globalement très proche de celle de $L G C$.

${ }^{25}$ C. Croizy-Naquet, op. cit., p. 248. Voir aussi plus largement sur le personnage de César les pages 243-249.
} 
traits négatifs du personnage. Comme l'a noté O. Collet, les deux textes, même s'ils sont globalement très proches, mériteraient peut-être une comparaison systématique, mais on ne l'entreprendra pas ici. Notons deux faits seulement, qui apparaissent d'ailleurs quelque peu contradictoires : d'un côté le recours à la prose et la réaffirmation, notamment dans les deux prologues, du lien avec Lucain, semblent impliquer un retour au modèle historique représenté par les Faits des Romains ; de l'autre, dans la continuité du processus engagé dans ce dernier texte, l'Hystore ajoute encore un peu à la revalorisation de César, notamment en atténuant certains passages négatifs du RJC, même si dans l'ensemble le personnage ne connaît pas de modification très sensible ${ }^{26}$.

Qu'on le situe donc par rapport à un modèle épico-romanesque ou par rapport à son prédécesseur immédiat, les Faits des Romains, qui avait déjà effectué un travail important dans ce sens, ce n'est pas finalement la revalorisation, certes incontestable, dont César est l'objet dans le RJC qui doit le plus surprendre: elle est essentiellement, selon nous, le produit de la transposition du personnage dans le contexte littéraire médiéval, avec la pratique traductive et le système des genres qui lui sont propres ; en somme Jean de Thuin n'a pas forcément cherché à réhabiliter César, il a d'abord voulu en faire un héros de roman, ce qui impliquait de toute manière sa revalorisation. Ce qui est beaucoup plus frappant en revanche, c'est bien la permanence chez le personnage ainsi transposé de plusieurs aspects négatifs contribuant à entretenir au moins son ambiguïté. La faute en est certainement à la représentation particulièrement dépréciative de Lucain, mais peut-être aussi, plus largement, à l'image de toute manière assez ambiguë de César au Moyen Âge, comme l'a bien montré J. Leeker ${ }^{27}$. Ainsi, au fil des adaptations successives, le personnage de César apparaît résister et conserver dans chacune de ses incarnations ses traits négatifs, et son caractère «problématique».

\section{César, un héros «problématique»}

Certes, si l'on en croit G. Lukacs, que César apparaisse comme un héros «problématique ${ }^{28}$ tend précisément à en faire un parfait personnage de roman. Il y a lieu néamoins de penser, selon nous, que c'est précisément ce caractère «problématique» qui a pu poser difficulté à un éventuel adaptateur de Lucain au $\mathrm{XII}^{\mathrm{e}}$ siècle et qui pourrait expliquer pourquoi il a fallu attendre si longtemps avant qu'un clerc se risque à faire de $L G C$ un roman, à la manière des Roman de Thèbes

\footnotetext{
${ }^{26}$ Tous les traits négatifs relevés plus haut sont conservés, à l'exception des quelques points suivants qui ne font pas, il faut le reconnaître, évoluer le personnage de façon bien significative : au moment du passage du Rubicon (p. 14-15), il n'est plus question de l'ire de César, à la différence du $R J C$ (v. 401 et 426) ; p. 20 le bellicisme de César n'est plus donné comme un trait permanent de son caractère $(R J C$, v. 605-606), mais comme la conséquence du discours de Curion; p. 34 et 147, il n'est plus question de l'orgueil de César (comparer avec RJC, v. 1148 et 5522); enfin p. 125 le jugement négatif porté sur la bataille de Pharsale n'incrimine plus, même indirectement, César (comparer avec RJC, v. 4615-4618).

${ }^{27}$ J. Leeker, Die Darstellung Cäsars in den romanischen Literaturen des Mittelalters, Francfort, Klostermann, 1986.

${ }^{28}$ Voir G. Lukacs, La Théorie du roman, Paris, Gonthier, 1975, p. 73 et suivantes.
} 
ou d'Eneas. Le succès probablement mitigé qu'a rencontré le $R J C$ en vers, comme sa rapide mise en prose le laisse soupçonner, fournit d'ailleurs un indice supplémentaire de cette difficulté, du problème que pouvait poser la construction de César en personnage de roman recevable par un public médiéval.

Premier problème qui se posait à un adaptateur de Lucain, la représentation très défavorable de César dans $L G C$, qui rendait à peu près impossible - comme le montre clairement le $R J C$ - l'élimination de tous ses aspects négatifs, qui en faisait forcément un personnage au moins ambigu, et donc délicat à manipuler. Il est vrai que le «héros problématique», pour ne pas être des plus courants dans la littérature médiévale (il suffit de penser au chevalier paré de toutes les vertus dans nombre de romans arthuriens), n'est pas non plus exceptionnel. Pour s'en tenir aux seuls romans antiques, Eneas, pourvu d'un portrait moral plutôt «contrasté ${ }^{29}$, du moins au départ, dans le Roman d'Eneas, et plus encore Alexandre, ce «héros ambivalent ${ }^{30}$, peuvent en offrir de bons exemples. Chez ces personnages cependant, qu'il s'agisse des textes latins ou de leurs adaptations françaises, les aspects négatifs sont loin d'être aussi marqués que dans le cas de César. Ainsi Eneas, s'il peut apparaître lâche et inconstant au début du roman qui lui est consacré, devient rapidement un héros pleinement positif. Quant à Alexandre, son cas est plus intéressant dans la mesure où il apparaît à bien des égards comparable à celui de César, ce qui permet une nouvelle fois de pointer le Roman d'Alexandre (dans la version d'Alexandre de Paris, la plus largement diffusée) comme le modèle romanesque le plus évident du RJC. Dès l'Antiquité et jusqu'au Moyen Âge, la réputation d'Alexandre, comme celle de César d'ailleurs, se construit en effet à travers des approches contrastées, des jugements contradictoires $^{31}$, dont le Roman d'Alexandre conserve notamment trace en opposant au modèle de prouesse, de largesse, de justice etc. qu'offre Alexandre l'orgueil démesuré dont il fait montre et qui causera finalement sa perte (comme le suggère Alexandre de Paris à la fin de son roman). Néanmoins la longue destinée littéraire qu'a connue Alexandre, depuis le Pseudo-Callisthène jusqu'à Albéric de Pisançon, Alexandre de Paris ou Thomas de Kent, a permis d'élever le conquérant à la stature de héros à la fois mythique et romanesque, et globalement d'en donner, surtout dans les romans en vers, une vision favorable; à l'inverse César, mis seulement au début du XIII ${ }^{\mathrm{e}}$ siècle sur le devant de la scène littéraire grâce aux Faits des Romains, de toute manière fortement marqué par la représentation dépréciative de Lucain, apparaît dépourvu d'une telle aura, reste somme toute un personnage plus historique que

\footnotetext{
${ }^{29}$ Cette appréciation se trouve dans A. Petit, «Eneas dans le Roman d'Eneas », Le Moyen $\hat{A} g e, 96,1990$, p. 67-79. On pourra aussi se reporter, sur le même personnage, à F. Mora, «Eneas est-il un héros épique? », Pris-ma, 9, 2, 1993, p. 229-244.

${ }^{30}$ Voir sur ce sujet les excellentes pages de L. Harf en introduction au Roman d'Alexandre, Paris, Le Livre de Poche, 1994, p. 43-53. Toujours sur l'ambivalence de l'Alexandre romanesque, on pourra aussi se reporter à F. Suard, «Alexandre est-il un personnage de roman ? », Bien dire et bien aprandre, 7, 1989, p. 77-87.

${ }^{31}$ On notera en passant que Lucain de son côté porte le jugement le plus défavorable sur le personnage d'Alexandre (voir le Livre X, v. 20 et suivants), ce qui n'est pas forcément une surprise, mais que cette vision du conquérant disparaît totalement dans le passage correspondant du RJC (v. 7149 et suivants).
} 
mythique, une figure où subsistent bien des traits négatifs, difficiles à concilier avec une existence de roman $^{32}$.

D'ailleurs, si l'on doit trouver quelques exemples de personnages assez comparables au héros du $R J C$, c'est plutôt du côté de la chanson de geste qu'il faudrait se tourner, et plus précisément vers le type du "héros démesuré » ${ }^{33}$, tel Girart de Roussillon, Ogier le Danois, Renaut de Montauban, plus encore Raoul de Cambrai, dont un certain nombre de traits ne sont pas sans rappeler le César mis en scène par Jean de Thuin. Dans Raoul de Cambrai en effet, on retrouve chez Raoul la même ambivalence, un semblable mélange de qualifications positives et négatives, associant aux vertus guerrières et chevaleresques non seulement l'orgueil et la desmesure, mais aussi l'ire, exactement comme chez César ${ }^{34}$ - ire qui peut apparaître comme le signe caractéristique de la violence meurtrière animant le personnage pendant presque toute sa carrière, violence allumée comme dans le $R J C$ suite à une injustice (le Vermandois refusé à Raoul, comme le triomphe refusé à César) et qui ne s'éteindra qu'à la mort du héros ${ }^{35}$. Ainsi il n'est pas interdit de penser, non pas que Jean de Thuin n'est pas parvenu à supprimer toute ambiguïté dans le personnage de César, mais qu'il n'a pas cherché à le faire ; qu'à côté du Roman d'Alexandre, qui lui offrait déjà un exemple intéressant de «héros ambivalent», l'auteur du RJC a pu aussi se donner pour modèle une chanson de geste mettant en scène quelque «baron révolté » et dont le fond de conflit intérieur n'est évidemment pas sans rapport avec $L G C$; enfin qu'il a peut-être voulu tenter au milieu du XIII ${ }^{\mathrm{e}}$ siècle une sorte de retour au plus ancien modèle épique, celui d'une chanson à caractère historique et à contenu exclusivement guerrier. En ce sens ce serait une tentative originale de se tenir au plus près du poème historique de Lucain, en visant la transposition dans la forme médiévale qui lui était logiquement la plus assimilable, la chanson de geste ; tentative trop originale même, comme en témoigne le rapide dérimage du $R J C$, qui laisse à penser qu'après les Faits des Romains la forme choisie par Jean de Thuin ne devait plus paraître en adéquation avec son objet.

Le second problème qui se posait à un adaptateur de Lucain, d'ailleurs lié au premier, tient plus précisément au facteur hiérarchique évoqué plus haut, au choix de

\footnotetext{
${ }^{32}$ La même conclusion ressort d'une comparaison entre Alexandre et César dans les Faits des Romains, où les deux conquérants apparaissent certes dotés pour bonne part des mêmes qualités et des mêmes défauts, mais où César reste tout de même marqué plus négativement qu'Alexandre. Voir C. Croizy-Naquet, «Alexander and Caesar in the Faits des Romains », The Medieval French Alexander, éd. D. Maddox et S. Sturm-Maddox, State University of New York, 2002, en particulier p. 172.

${ }^{33}$ Sur ce type de personnage, voir surtout M. de Combarieu, L'Idéal humain et l'expérience morale chez les héros des chansons de geste (des origines à 1250), Université de Provence, 1979, p. 628-664, mais aussi W. Calin, The Old French Epic of Revolt, Genève, Droz, 1962.

${ }^{34}$ Voir Raoul de Cambrai, trad. W. Kibler d'après l'éd. de S. Kay, Paris, Le Livre de Poche, 1996, notamment les vers 505, 682, 1014, 1272, 1284, 1296, 1406, 2155, 2739, 2809, 2867 et 2919.

${ }^{35}$ Sur ces aspects du personnage de Raoul, voir, en plus des ouvrages cités ci-dessus, É. Baumgartner et L. Harf-Lancner, Raoul de Cambrai : l'impossible révolte, Paris, Champion, 1999, p. 95-117 et M. Botero Garcia, «Le personnage de Raoul dans Raoul de Cambrai ou le fatum héroïque d'un chevalier démesuré », Cahiers de recherches médiévales, 6, 1999, p. 111-122.
} 
faire d'un personnage important parmi d'autres dans $L G C$ le héros d'un récit tout entier centré sur lui. En fait, pour le traitement hiérarchique du personnage de César, deux voies étaient possibles, déjà ouvertes par les romans antiques du XII ${ }^{\mathrm{e}}$ siècle adaptant des textes latins, la première majoritaire, plus évidente, mais qui n'était pas la plus facile à suivre à partir de $L G C$, l'autre minoritaire et somme toute assez particulière.

La première voie, qui est empruntée dès les premiers romans antiques et qui apparait comme très largement dominante pendant tout le Moyen Âge, et qui de ce fait était la plus à même de s'imposer à un adaptateur de Lucain, c'est celle du roman à héros unique. C'est cette voie qu'ont suivi le Roman d'Alexandre, dans toutes ses versions depuis Albéric de Pisançon, le Roman d'Apollonius de Tyr ou le Roman d'Eneas, et bien d'autres romans eux; voie suivie d'autant plus facilement, même par un personnage relativement ambivalent comme Alexandre que, comme on l'a déjà suggéré plus haut, chacun de ces romans pouvait s'appuyer sur des textes latins déjà pourvus d'un héros nettement identifié et plus ou moins teinté de romanesque. On se trouve là dans un cas où la structure narrative et le système des personnages de l'œuvre source sont globalement assimilables par l'œuvre cible, sans discordances majeures, ce qui n'est évidemment pas le cas du $R J C$ par rapport à $L G C$, dont il bouleverse profondément la hiérarchie des personnages.

Il existait cependant au XII ${ }^{\mathrm{e}}$ siècle une seconde voie, représentée seulement par le Roman de Thèbes et de Troie, celle du roman à personnages principaux multiples, donc sans véritable héros. Comme l'a bien montré en effet A. Petit en comparant ces deux derniers romans au Roman d'Eneas, la structure narrative qu'ils présentent leur est tout à fait particulière :

Le Roman d'Eneas diffère profondément de Thèbes et de Troie pour ce qui est de la conjointure. Ces deux romans sont l'histoire d'affrontements collectifs aux héros multiples, qui se terminent par l'anéantissement de la plupart des combattants et celui d'une cité. Dans le Roman d'Eneas, un héros principal, sinon unique, accomplit sa destinée à l'occasion de laquelle éléments amoureux et éléments guerriers finissent s'interpénétrer totalement. ${ }^{36}$

Le choix de cette voie est à remarquer, car il n'est pas des plus fréquents dans la littérature médiévale. En ce qui concerne le Roman de Thèbes et de Troie, au moins deux raisons permettent de l'expliquer. D'abord le fait que La Thébaïde de Stace, comme $L G C$, ou les deux résumés tardifs de Dictys et Darès utilisés par Benoît de Sainte-Maure présentaient une structure similaire sans héros central, qu'il s'agissait seulement de pouvoir reproduire et de choisir de reproduire. Choix qui peut trouver son explication dans un second fait, la vocation un peu particulière de ces textes, s'inscrivant dans une perspective idéologique et politique spécifique, celle de conférer à la dynastie Plantagenêt le prestige de prétendues origines troyennes. Ainsi importait-il peut-être peu finalement que le Roman de Thèbes et de Troie soient dépourvus de véritable héros, étant donné leur portée d'abord historique qui les destinait, en les associant au Roman d'Eneas, à donner aux rois d'Angleterre une glorieuse préhistoire que le Roman de Brut et le Roman de Rou de Wace (ou la

${ }^{36}$ A. Petit, Naissances du roman, p. 497. 
Chronique des ducs de Normandie de Benoît de Sainte-Maure) devaient se charger de poursuivre et de mener jusqu'au temps d'Henri II Plantagnêt ${ }^{37}$.

Pour un clerc envisageant de traduire Lucain au $\mathrm{XII}^{\mathrm{e}}$ siècle, puisque $L G C$ est une épopée sans héros, possédant plusieurs personnages centraux, le choix de la seconde voie, à la façon de l'auteur du Roman de Thèbes adaptant Stace, apparaissait donc à la fois comme le plus logique et comme le moins évident, étant donné le caractère dominant de la première voie dans les habitudes narratives médiévales; ainsi le problème posé par l'ambivalence de César se doublait-t-il d'un second, celui d'en faire le héros d'un roman. Si l'on considère en outre que le choix un peu particulier des Roman de Thèbes et de Troie a pu être guidé par des considérations politiques et idéologiques, il apparaît enfin que le personnage de César pouvait poser un troisième problème, du moins dans le cadre d'une production littéraire dépendante du milieu Plantagenêt. On a dit que les Roman de Thèbes, de Troie et d'Eneas étaient probablement liés dans leur conception à des intentions de glorification, en remontant jusqu'aux soi-disant origines troyennes des rois d'Angleterre. Certes César aussi prétendait à de telles origines, comme le rappelle d'ailleurs le RJC (v. 5932, en parfaite contradiction avec $L G C$, Livre III, v. 212-213), et la gloire du conquérant romain aurait pu après tout s'ajouter à celle des Plantagenêt, en intercalant son histoire quelque part entre le Roman d'Eneas et le Roman de Brut. Et de fait le général romain fait son apparition chez Wace, où il bénéficie d'ailleurs d'un traitement beaucoup plus favorable que chez Geoffroy de Monmouth, notamment grâce au portrait suivant qui marque son entrée dans l'histoire bretonne :

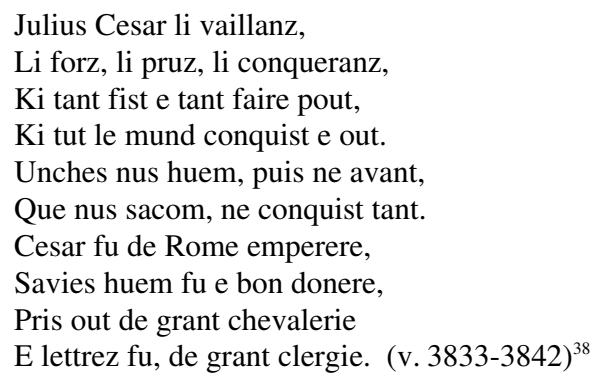

Pour le reste, et en dépit de leurs communes origines troyennes (voir les v. 38703873), César est avant tout le premier envahisseur des Bretons, celui qui leur a imposé le tribut de Rome, inaugurant ainsi les relations complexes et souvent hostiles entre les deux pays, et somme toute un ennemi de l'Angleterre. L'éloge vaut surtout pour souligner à rebours la valeur des Bretons qui dans un premier temps ont été capables de résister à un tel conquérant, sans qu'il s'agisse de lui rendre plus qu'un

\footnotetext{
${ }^{37}$ La relation étroite qui unit ces différents romans probablement tous issus du même milieu Plantagenêt se trouve confirmée dans le fait qu'on les trouve souvent associés dans les mêmes mss. ; à l'inverse il est à noter que les trois mss. qui nous ont conservé complet le RJC ne contiennent que ce seul roman.

${ }^{38}$ Wace, Roman de Brut, éd. et trad. J. Weiss, University of Exeter, 1999.
} 
rapide hommage ${ }^{39}$. César, ennemi des rois bretons, cela pourrait donc faire une raison supplémentaire pour que les clercs œuvrant à la cour d'Henri II Plantagenêt - où s'est développée, dans la seconde moitié du XII ${ }^{\mathrm{e}}$ siècle, "une véritable école d'imitation de l'Antiquité ", selon le mot de C. de Boer ${ }^{40}-$ n'aient pas envisagé d'adapter le texte de Lucain, que pourtant ils devaient certainement connaître.

Il est évidemment difficile, en fin de compte, de savoir précisément pourquoi Lucain n'a pas été mis en roman avant la seconde moitié du XIII ${ }^{\mathrm{e}}$ siècle. On peut supposer que sont entrés en jeu plusieurs facteurs liés à la fois au texte de Lucain et au personnage de César. De fait il nous semble que $L G C$ présentait au moins une triple difficulté : un texte inachevé, qu'il fallait compléter (avec d'autres textes peutêtre moins accessibles, par exemple ceux du corpus césarien); une représentation particulièrement défavorable de César ; une structure narrative à héros multiples, qui appelait à suivre pour l'adaptation une voie minoritaire dans le roman du XII ${ }^{\mathrm{e}}$ siècle, celle du Roman de Thèbes et du Roman de Troie, et qui entrait en outre dans un cadre idéologique où César ne pouvait guère avoir sa place. On peut en fait penser que la notoriété de la figure de César au Moyen Âge tendait à empêcher de ne pas en faire le héros (et non un personnage parmi d'autres) dans un récit ayant fait le choix le lui consacrer une certaine place (comme peuvent en témoigner à leur manière les Faits des Romains, finalement consacrés au seul César), en même temps que l'ambiguité, l'ambivalence toujours inhérente à la représentation médiévale du personnage rendaient particulièrement problématique sa mise en place en tant que hé$\operatorname{ros}^{41}$. Dans ces conditions, on comprendra peut-être qu'au XII ${ }^{\mathrm{e}}$ siècle le poème de Lucain ait pu décourager l'adaptation ${ }^{42}$.

Silvère Menegaldo Université d'Orléans

\footnotetext{
${ }^{39}$ Sur la figure de César dans le Roman de Brut de Wace (et sa source latine, l'Histoire des rois de Bretagne de Geoffroy de Monmouth), voir notamment J. Leeker, op. cit., p. 175-185 et L. Mathey-Maille, «Mythe troyen et histoire romaine : de Geoffroy de Monmouth au Brut de Wace », Entre fiction et histoire : Troie et Rome au Moyen Âge, éd. É. Baumgartner et L. Harf, Presses de la Sorbonne Nouvelle, 1997, p. 113-125.

${ }^{40}$ Cité par F. Mora dans l'introduction à son édition du Roman de Thèbes, Paris, Le Livre de Poche, 1995 , p. 7.

${ }^{41}$ L'ouvrage déjà cité de J. Leeker met bien en évidence à la fois l'importance de la figure de César au Moyen Âge et le caractère presque toujours ambigu des représentations dont il est l'objet, par exemple dans les chroniques locales françaises, où il prend l'apparence, dans le cadre de la guerre des Gaules, «eines halb gehaßten und halb bewunderten Aggressors » (p. 156).

${ }^{42}$ La version définitive de cet article a grandement bénéficié des remarques et suggestions de Mme Szkilnik et de MM. Duval et Ribémont, auxquels je tiens à adresser tous mes remerciements.
} 